\title{
Novel aberrant nuclear behavior in abortive conjugation of Tetrahymena thermophila: Joint selection of a meiotic product and macronucleus during nuclear selection
}

\author{
Atsushi Mukai and Hiroshi Endoh* \\ Department of Biology, Faculty of Science, Kanazawa University, \\ Kanazawa 920-1192, Japan
}

(Received 11 February 1999, accepted 13 April 1999)

\begin{abstract}
In conjugation of Tetrahymena thermophila, the paroral zone, cortical cytoplasm in the vicinity of the cytostome, is the site where nuclear selection occurs; one of the four meiotic products is selected in this site prior to the production of gametic pronuclei. During inbreeding cross experiments, several sterile strains were obtained which showed aberrant nuclear behavior. Conjugants of these strains normally underwent meiosis, resulting in the generation of four meiotic products. They, however, failed to complete the process of nuclear selection and aborted the subsequent conjugation sequences. During nuclear selection, macronucleus was frequently selected instead of a meiotic product. A novel aberrant nuclear behavior was observed: Macronucleus and a meiotic product were jointly selected and the both nuclei simultaneously attached to the same paroral zone. When this simultaneous attachment occurred in one partner cell of a pair, nuclear selection was never observed in the other partner cell. This result suggests that a conjugating pair has only two attachment sites on the paroral zone during nuclear selection, and that the distribution of the sites is occasionally distorted in abortive conjugation.
\end{abstract}

Conjugation in Tetrahymena thermophila is initiated by an interaction of sexual cells with different mating types. Conjugating cells undergo meiosis and the subsequent developmental process. The process consists of a series of nuclear events such as nuclear selection after meiosis, postmeiotic division to produce gametic nuclei, nuclear exchange, fertilization, and postzygotic development including nuclear differentiation (Orias, 1986). It is known that aberrant conjugation, which shows highly anomalous nuclear behavior, rarely occurs in crosses between young fertile strains, while it does frequently in crosses between ageing clones (Weindruch and Doerder, 1975; Schlonick and Bruns, 1982). It has been also reported that UV irradiation at meiotic prophase induces a similar abnormal conjugation (Kobayashi and Endoh, 1998). Such aberrant conjugation is referred to as abortive conjugation which is characterized by the failure of nuclear selection after meiosis as well as the abortion of the further development in conjugation.

Nuclear selection occurs at the paroral zone i.e., cortical

\footnotetext{
* Corresponding author.
}

cytoplasm in the vicinity of the cytostome. Following the selection of one meiotic product out of four, the selected nucleus attaches to the paroral zone where is protected from degradation. Then it undergoes mitosis to produce gametic pronuclei. The three unselected meiotic products become pyknotic and disappear later on. In contrast to the normal positioning of a meiotic product, there are cases of abortive conjugation, in which macronucleus is selected instead of a meiotic product. In these abnormal cases, the selected macronucleus attaches to the paroral zone during nuclear selection (Nanney and Nagel, 1964; Gaertig and Fleury, 1992). With regard to nuclear selection, the researchers of the field have an agreement that only when a meiotic product failed to attach to the paroral zone, the macronucleus is reluctantly selected, regardless of the nature of nuclei.

During our inbreeding cross experiments, several completely-sterile progenies were produced. These progenies showed aberrant nuclear behavior as in the abortive conjugation mentioned above. In the current paper, we describe a novel aberrant nuclear behavior in which macronucleus and a meiotic product were jointly selected 
only in one partner cell of a pair, and as a result, both nuclei attached to the same paroral zone during nuclear selection.

All strains used in this study belong to T. thermophila. Original strains, WA6 and WD6, were kindly supplied by Sugai (Ibaraki university, Japan). Cell cultivation and induction of conjugation have been described elsewhere (Haremaki et al., 1996; Kobayashi and Endoh, 1998). Conjugation was induced by mixing equal numbers of cells with the different mating types at $26^{\circ} \mathrm{C}$, and the cells were fixed with formalin (final 3\%) every one hour. The fixed cells were stained with $1 \mu \mathrm{g} / \mathrm{ml}$ DAPI (4,6-diamidino-2phenylindole dihydrochloride) and observed under fluorescence microscope.

In cross experiments, exconjugants were separated into culture medium, and after their first fission, four cell lines (caryonides) which were derived from a single pair were separated again into culture medium. Further inbreeding cross experiments were performed between these sister caryonides of different mating types.

Strains used in this study were obtained during the following process. Starting from the cross between the parental strains, WA6 and WD6, three successive inbreeding crosses were carried out. Fertile strains, W4K2 and W4J9, produced in $\mathrm{F}_{2}$ showed normal phenotype and were used as a control. When they were crossed to produce $\mathrm{F}_{3}$ progeny, the sister-caryonidal strains, W5D6 and W5G6, used mainly in this study were obtained first. They were characterized by their complete sterility: So far we could not obtain any true $\mathrm{F}_{4}$ progeny, although more than 400 clones were isolated. Cytological observations revealed that the process of conjugation in these sterile strains normally proceeded until the completion of meiosis, where four haploid meiotic products were generated. In conjugation of the fertile strains, a meiotic product attaches to the paroral zone during nuclear selection (Fig. 1A). In the sterile strains, however, several kinds of abnormalities were observed. The most frequent abnormality was that the macronucleus behaved as if it were the selected meiotic product. After a micronucleus underwent meiosis, the macronucleus elongated and occupied the paroral zone in both partners of conjugants (Fig. 1B). Although there were a few meiotic products located in the vicinity of the paroral zone, they never attached to the zone. It was also rarely observed that the micronucleus in one partner cell of a pair and the macronucleus in the other partner attached to the paroral zone (data not shown). A novel abnormal nuclear behavior was observed: Both macronucleus and a meiotic product were jointly selected and they simultaneously attached to the same paroral zone (Fig. 1C, D). In these cases, the joint nuclear selection occurred only in one partner cell of the pair, and the other partner always failed to select any nucleus. Our observation suggests that the paroral zone can anchor two nuclei in a pair, and that the distribution of the anchor
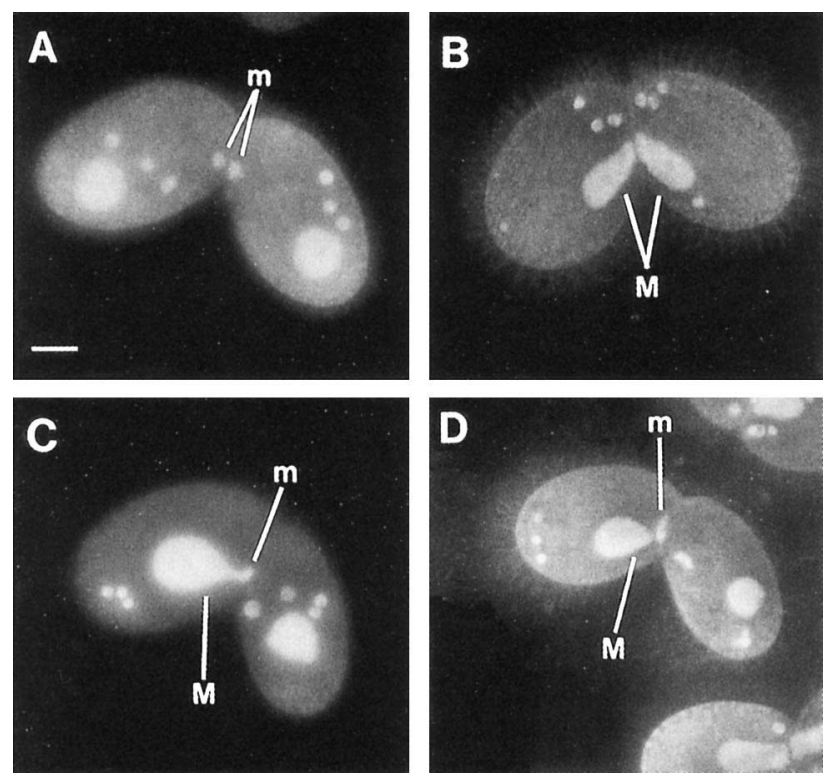

Fig. 1. Fluorescence microscopy of DAPI-stained nuclei at the stage of nuclear selection during conjugation. Note that each cell in a pair has one macronucleus and total of four haploid micronuclei. A) Conjugation in wild type. One of the four meiotic products was selected, resulting in the attachment to the paroral zone in both cells of the pair. B-D) Conjugation in the sterile strains. B: The macronucleus attaches to the paroral zone in both cells of a pair instead of the micronucleus. The micronuclei located in the anterior region do not attach to the zone. C: The macronucleus and the postmeiotic micronucleus simultaneously attach to the paroral zone in one partner cell of a pair (left). Four unselected micronuclei fail to attach to the paroral zone in the other partner cell (right). D: The simultaneous attachment is also shown in one partner cell (left). In the other partner cell (right), three unselected micronuclei located in the posterior region have been pyknotic, judging from their sizes. The remaining micronucleus located near the paroral zone also fails to attach to the zone. Abbreviation. M: Selected macronucleus. $\mathrm{m}$ : Selected micronucleus. Bar in the panel A indicates $10 \mu \mathrm{m}$.

sites on the paroral zone is rarely distorted in the abortive conjugation.

When conjugation was induced between the sterile strains $\left(\mathrm{F}_{3}\right)$, W5D6 and W5G6, macronuclear selection occurred at high frequencies, whereas normal nuclear selection was also observed at relatively low frequencies, as shown in Table 1. Macronuclear selection showed a peak $9 \mathrm{~h}$ after mixing different mating types. In contrast, the percentage of the conjugants which underwent normal nuclear selection showed a peak earlier and had decreased at this time. Given these observations, the process of macronuclear selection might last longer than that of normal nuclear selection. Table 1 also shows that the joint selection did not last longer. Thus, when at least one meiotic product is selected, the process of nuclear selection seems to complete for a short time, as in the normal process. After pair separation, exconjugants had retained four micronuclei and a parental macronucleus even in so later stage and reconjugated (data not shown), 
Table 1. Percentage of conjugants with normal and abnormal nuclear behavior during nuclear selection in conjugation of the sterile strains

\begin{tabular}{|c|c|c|c|c|}
\hline \multirow[t]{2}{*}{ Selected nuclei } & \multicolumn{4}{|c|}{$\begin{array}{c}\text { Time after mixing different } \\
\text { mating types }(\mathrm{hrs})\end{array}$} \\
\hline & 7 & 8 & 9 & 10 \\
\hline Meiotic product (Normal) & 15 & 16 & 5 & 0 \\
\hline Macronucleus ${ }^{1)}$ & 10 & 38 & 54 & 18 \\
\hline $\begin{array}{l}\text { Meiotic product and } \\
\text { macronucleus }{ }^{2} \text { (Joint } \\
\text { selection) }\end{array}$ & $0.1<^{3)}$ & 0 & 0 & 0 \\
\hline
\end{tabular}

Note: Conjugation was induced between the sterile strains, W5D6 and W5G6. At each time, more than 2,000 conjugants were counted.

1) Macronuclear selection occurs when macronucleus attached to the paroral zone (See Fig. 1B).

2) Joint selection occurs when macronucleus and a meiotic product jointly attached to the paroral zone in one partner cell and at the same time, any nucleus failed to attach to the zone in the other cell of a pair (See Fig. 1C, D).

3) There were many cases which possibly suggest occurences of joint selection. However, indecisive instances except for the evident ones, as shown in Fig. 1, were not counted as the joint selection.

as in round 2 genomic exclusion (Bruns, 1986). Judging from the sizes, the four nuclei might be the haploid meiotic products. These results suggest that four meiotic products were free from pyknosis after meiosis and the subsequent conjugation process was aborted after the failure of normal nuclear selection.

Normal nuclear selection of a meiotic product in conjugation of the sterile strains was also observed, as mentioned above. Even in such cases, however, neither synkaryon formation (fertilization) nor the subsequent process including development of macronuclear anlagen were observed (data not shown). These results suggest that the sterile strains have a defect which is responsible for the abortion of the subsequent conjugation process and the incapability to produce the $\mathrm{F}_{4}$ progeny, regardless of the success or failure of nuclear selection. On the other hand, when the sterile strain was crossed with fertile (normal) one, no conspicuous anomaly was detected (data not shown). When either of conjugating cells is fertile, conjugation normally proceeds. Probably a product necessary for normal conjugation would be transferred into a defective partner through fine cytoplasmic bridges and it would repair the defect in the sterile strain.

Microtubules are known to be involved in the intracellular positioning of nuclei. Gaertig and Fleury (1992) showed in detail the distribution patterns of intracytoplasmic microtubules during conjugation in Tetrahymena utilizing the immunofluorescence method. Their observations are as follows: Microtubular bundles between the paroral zone and the selected meiotic product are formed only when the nucleus is functional. In abortive conjugation, there are bundles which linked the selected macronucleus and the paroral zone. When one of the meiotic products fails to become a functional nucleus, the microtubular bundles from the paroral zone might anchor the macronucleus. Considering their observations, the following interpretation of the result of our experiment is possible: The bundles which failed to anchor not only a meiotic product but also macronucleus in one partner cell of a pair moved to the other partner cell, resulting in the joint selection in the latter cell.

The result of our experiment deserves an alternative interpretation. The selected meiotic product usually undergoes prezygotic division to produce two gametic pronuclei, i.e., migratory and stationary nuclei. Conjugants exchange the migratory nucleus each other and then nuclear fusion (karyogamy) occurs. According to Gaertig and Fleury's observation, again, two pronuclei are anchored by microtubules from the paroral zone after nuclear transfer and they in turn proceed to karyogamy. The aberrant nuclear behavior presented in this paper may reflect on the nature of this karyogamy stage. If a small fraction of conjugants already go into the transfer and karyogamy stages, they should have an ability to anchor two pronuclei. Therefore, in the joint selection presented here, two different kinds of nuclei, macronucleus and a meiotic product, could have been selected so that they were anchored instead of the two pronuclei. However, the two nuclei could not fuse each other due to the difference of their intrinsic nature. The failure of karyogamy might have arrested the subsequent progress of the conjugation sequences.

We express our special thanks to T. Sugai for helpful discussions and a kind supply of the original stocks used in this study. We also thank M. Saiki for her critical reading of the manuscript and encouraging suggestions.

\section{REFERENCES}

Bruns, P. J. (1986) Genetic organization of Tetrahymena. In: The Molecular Biology of Ciliated Protozoa (ed.: J. G. Gall), pp 27-44. Academic Press, New York.

Gaertig, J., and Fleury, A. (1992) Spatio-temporal reorganization of intracytoplasmic microtubules is associated with nuclear selection and differentiation during the developmenral process in the ciliate Tetrahymena thermophila. Protoplasma 167, 74-87.

Haremaki, T., Sugai, T., and Takahashi, M. (1996) Involvement of active cellular mechanism on the disorganization of oral apparatus in amicronucleate cells in Tetrahymena thermophila. Cell Struct. Funct. 21, 73-80.

Kobayashi, T., and Endoh, H. (1998) Abortive conjugation induced by UV-B irradiation at meiotic prophase in Tetrahymena thermophila. Dev. Genet. 23, 151-157.

Nanney, D. L., and Nagel, M. J. (1964) Nuclear misbehavior in an aberrant inbred Tetrahymena. J. Protozool. 11, 465-473.

Orias, E. (1986) Ciliate conjugation. In: The Molecular Biology of Ciliated Protozoa (ed.: J. G. Gall), pp 45-84. Academic Press, New York. 
Schlonick, S. B., and Bruns, P. J. (1982) Conditional lethality associated with macronuclear development in Tetrahymena thermophila. Dev. Biol. 93, 216-225.
Weindruch, R. H., and Doeder, F. P. (1975) Age-dependent micronuclear deterioration in Tetrahymena pyriformis, syngen 1. Mech. Ageing Dev. 4, 263-280. 